Military Technical College Kobry El-Kobba

Cairo, Egypt

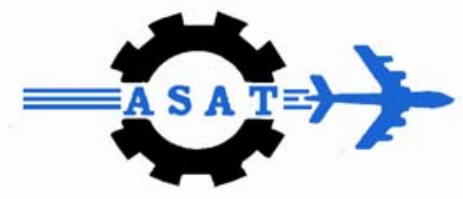

12-th International Conference

on

Aerospace Sciences \& Aviation Technology

\title{
DESIGN AND IMPLEMENTATION OF A POWER SYSTEM PHASOR MEASUREMENT UNIT
}

\author{
A. E. Eliwa *
}

\section{ABSTRACT}

In large power systems, the transmission lines, generators and loads are considered to be continuous functions of position and time (space). The application of the limit of zero generator internal impedance, with finite rotor inertia and zero transmission line impedance per unit length yields a nonlinear partial differential equation in time and two spatial dimensions for the rotor power angle. This model is called continuum model. Any disturbance in the system implies to electromechanical wave propagation in the system. Not only the models or simulations are sufficient for describing the power system electromechanical wave propagation; where this phenomenon needs a practical proof. The phasor measurement unit (PMU) can do that. In this paper; a complete design and realization of a PMU is implemented to detect the phenomenon. The PMU was implemented in Electrical Machines Lab. in Military Technical College, Cairo, Egypt. A photograph collection album for the preceding PMU and its laboratory realization, are presented in the figures of this paper. Also, the overall PMU circuit diagram with its components is placed in the paper articles.

\section{KEYWORDS}

Power system, phasor measurement unit, and PMU.

\footnotetext{
* Egyptian Armed Forces
} 


\section{INTRODUCTION}

Modern electric utility control centers use state estimators to monitor the state of the power system. The state estimator uses various measurements (such as complex powers, voltage and current magnitudes) received from different substations, and through an iterative nonlinear estimation procedure, calculates the power system state. The state (vector) is a collection of all the positive sequence voltage phasors of the network and from the time the first measurement is taken to the time when the state estimate is available, several seconds or minutes may have elapsed [1].

Because of the time skew in the data acquisition process, as well as the time it takes to converge to a state estimate, the available state vector is at best an averaged quasi-steady-state description of the power system. Consequently, the state estimators available in present-day control centers are restricted to steady-state applications only [2].

In power system stability studies it is accepted that the internal e. $\mathrm{m}$. f. of a synchronous machine is in phase coincidence with the quadrature axes of the rotor. The phasor measurement unit (PMU) is defined as the phase angle between the terminal voltage and induced e. $\mathrm{m}$. $\mathrm{f}$. of the machine. The value of the power angle, which changes in both steady state and transient conditions, are most important merits of stable operation of synchronous machines [3].

Actual measurements of power angle and speed error are necessary for both control and measurement purposes. The stroboscopic method of power angle measurement is quite successful at steady state conditions. This method is simple, accurate, and inexpensive. On the other hand it is not suitable for voltage regulators and speed governing system. Tachogenerator is usually used as a sensor to produce voltage signal proportional to rotor speed, since their mechanical assembly requires certain arrangement; optical transducers are preferred for rotor position detection [4].

Using an infrared optical transducer, which is not affected by ambient light, senses the rotor position. It transmits an infrared beam to the rotor axis and then the reflected pulses (which represent the rotor position) and the signal, which represents the infinite bus voltage. The phase difference is then converted to a DC voltage level proportional to the magnitude of the power angle.

Also, this paper presents a rotor speed-measuring device, which counts the reflected pulses from the rotor axis and converts this count to DC voltage level proportional to the rotor speed. The tests and calibration results show that the device is accurate and simple and it can be used for both measuring and control purposes [5].

As a new trend, phasor measuring units (PMUs) using synchronization signals from the GPS (Global Positioning System) satellite system has evolved into mature tools and is now being manufactured commercially. The GPS receiver provides the 1 pulse-per-second (pps) signal and a time tag, which consists of the year, day, hour, minute, and second. The time could be the local time, or the UTC (Universal Time Coordinated). The I-pps signal is usually divided by a phase-locked oscillator into the required number of pulses per second for sampling of the analog signals. In most systems being used at present, this is 12 times per cycle of the fundamental frequency. The analog signals are derived from the voltage and current transformer secondary, with appropriate anti-aliasing and surge filtering [3]. 


\section{OPTICAL TRANSDUCERS}

The optical transducer is used to convert the revolutions of the machine into train of pulses whose rising edges are coincident with the quadrature axis of the field winding, added with it some error. The repetition rate of these pulses represents the rotor speed. It is composed of an infrared transmitter, infrared receiver and shaping circuit. These components are assembled together on a $2.5 \times 6 \mathrm{~cm}$ printed circuit board and inserted into a cylindrical probe with $2.5 \mathrm{~cm}$ diameter and $7 \mathrm{~cm}$ long. A holder at a right angle to the rotor axis fixed the probe to the machine. A fivecentimeter length of the rotor axis in front of the optical transducer probe is painted with white colour (reflecting surface), and a thin black strip (absorbing surface) with one centimeter wide is fixed on the white surface of the rotor axis. When the machine runs, the white and black areas are exposed alternatively to the transmitted infrared beam. The numbers of black strips on the rotor are equal to the number of pole pairs of the synchronous machine under consideration. In this work the number of pole pairs is one, so only one black strip will be fixed on the rotor. When the black strip is under the probe, it interrupts the reflected infrared beam and the electronic circuit in the probe generates a pulse, the leading edge of which is synchronized with the leading edge of the black strip. The duration of this pulse depends on the width of the black strip as well as the speed of the rotor.

The circuit diagram of the optical transducer is shown in figure (1). Infrared diode D1 is used to generate a beam of continuous wave of the infrared band towards the shaft of the rotor. If reflecting surface (white area) is in front of the probe (during the rotation) the reflected wave will illuminate the phototransistor TR1. This transistor is sensitive to the infrared wave band and will conduct to pass small amount of current. This is amplified by transistor TR2. The amplified current passes through resistor R3 that acts as emitter load for TR2. The voltage generated across R3 is applied to inverted input of the comparator COMP1. The non-inverted input of COMP1 is biased to some positive voltage, which can be adjusted by potentiometer VR1.

The normal operation VR1 is adjusted such that the voltage drop across R3 is larger than that across VR1. Therefore, the output voltage of the comparator in this case is near zero voltage. Transistor TR3 in this case is turned off and a zero voltage appears across emitter resistor R6. The L.E.D., which is connected to the collector of TR3, will be in off state. When the probe is in front of the black strip, no light will be reflected on phototransistor TR1. And therefore the voltage drop across R3 is zero voltage and hence the comparator COMP 1 will generate a pulse. Transistor TR3 will be turned on and a pulse appear across R5 in this case the L.E.D. will be lighted. This sequence is repeated during the rotation of the rotor.

\section{POWER ANGLE MEASURING DEVICE}

The block diagram for the power angle $(\delta)$ measurement is shown in figure (2). This device is fed by two signals; the first is obtained from phase voltage of the infinite bus. It is stepped down and converted to square wave of the same frequency by the zero crossing detector. The second signal is generated from the rotor optical transducer. The pulse width obtained is $2.5 \mathrm{~ms}$ when a black strip of one centimeter wide is used and at rotor speed of 3000 r.p.m. The output signal from zero crossing detector circuit and the output of optical transducer are fed to the phase detector 
circuit, which works as a bistable multivibrator.

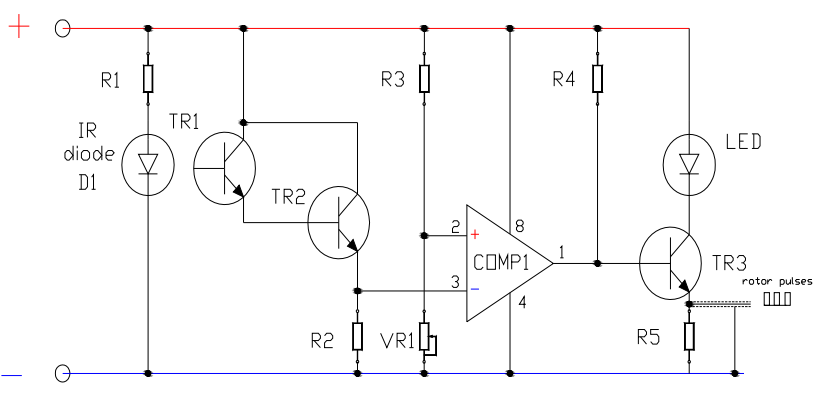

Fig. 1. Circuit diagram of the optical transducer

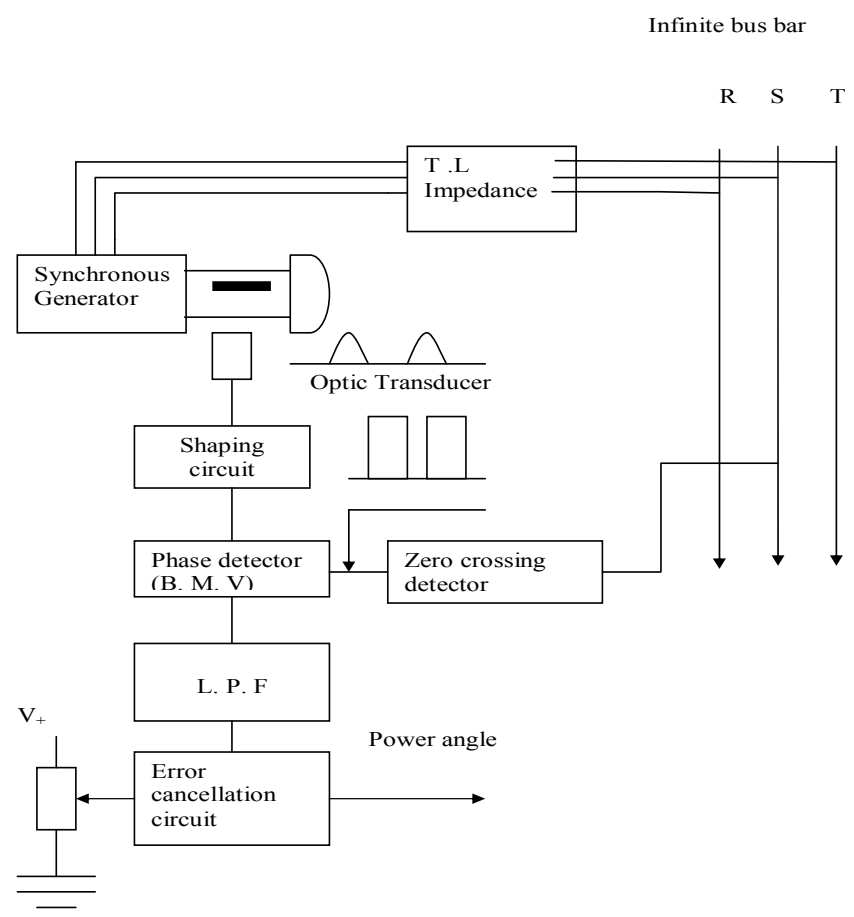

Fig. 2. Block diagram for forming the power angle

The output of this stage is a train of pulses whose frequency is the supply frequency and with a pulse width equals to the phase difference between the infinite bus voltage and the pulsed waveform generated from the rotor optical transducer. These pulses are then converted into a DC voltage level by using active low pass filter (L.P.F). The resulting DC voltage is proportional to the exact value of the power angle $(\delta)$, with some error signal $(E)$, which arising from the random attachment of the black strip on the rotor axis. An error cancellation circuit is used to eliminate the value of the error voltage. The obtained DC voltage can be used for measuring or recording purposes by connecting this signal for example to an oscilloscope or $x-y$ plotter to monitor the steady state and dynamic performance of the synchronous machines. The DC voltage representing the power angle can also be used for control purposes. Using this signal as a stabilizing signal to be used in conjunction with automatic voltage regulators or speed governing systems can do this. The complete circuit diagram of the power angle device is shown in 
figure (3) the circuit diagram of each block in the figure will be explained in the following sections.

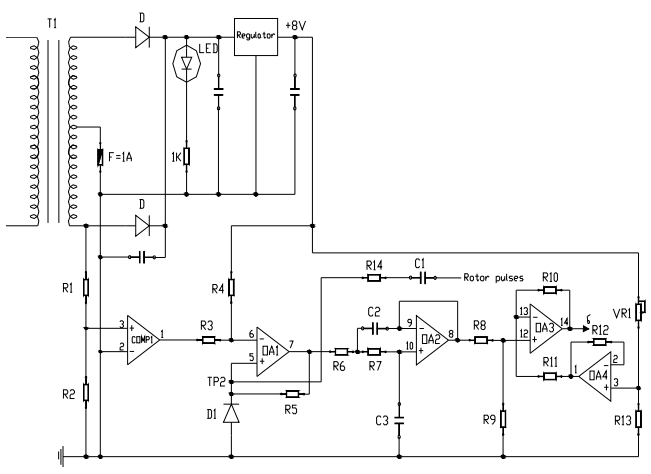

Fig. 3. Circuit diagram of the power angle device

\subsection{Zero crossing detector circuit}

The circuit diagram of the zero crossing detector is composed of, a step-down transformer T1, comparator COMP1, resistors R1 and R2. Infinite bus voltage is stepped-down to $6 \mathrm{~V}_{\text {rms }}$ by the transformer T1. The resistors R1 and R2 are used as a potential divider, which attenuates the stepped sine wave voltage to about 1 $V_{\text {rms. }}$ This voltage is applied to the non-inverting input of the comparator COMP1, while the other input of the comparator (inverting-input) is grounded. During the positive half cycle of the attenuated sine wave the non-inverting input of COMP1 is more positive than the inverting input, so the output of the comparator is saturated to high level positive direction. During the negative half cycle of the attenuated sine wave the output of the comparator will be zero potential. So the output of the comparator is a square wave pulses whose width is equal to the half wavelength (10 ms for $50 \mathrm{~Hz}$ operations). The function of the comparator in this circuit is to detect the instant of crossing the time axis by the attenuated sine wave and converts it to square pulses during the positive half cycles.

\subsection{Phase detector circuit}

The circuit diagram of the phase detector (bistable multivibrator) is composed of an operational amplifier OA1, resistors R3, R4, R5, R14, condenser C1 and diode $\mathrm{D} 1$. The rotor pulses from optical transducer are differentiated by the series circuit R14 and C1. The differentiated positive pulses are applied to the non-inverting input of the OA1. While the negative differentiated the voltage drop of the diode D1 limits pulses. During the negative half cycle of the infinite bus voltage the output of the comparator COMP1 is low and the resistors R3 and R4 work as a potential divider. The resistor R3 is chosen at smaller value than that of R4, so that a small positive voltage is applied to the inverting input of OA1. This voltage is less than the amplitude of the positive edge of the differentiated pulse so the output of OA1 is jumped to the positive saturation level. The feedback resistor R5 maintains the output of OA1 in saturation condition.

When the trailing edge of the infinite bus voltage signal is applied to the noninverting input of COMP1, its output will jump to positive saturation. This voltage is 
applied to the non-inverting input of OA1, so its output falls to the zero potential. Hence a positive pulse is obtained from the output of the OA1, whose leading edge coincides with the positive edge of the rotor pulse and its trailing edge coincides with the positive edge of the infinite bus voltage as shown in figure (4). The pulse width obtained from OA1 represents the phase difference between rotor pulse and infinite bus voltage, which corresponds to the power angle. The output pulse from the phase detecting circuit is fed to an active low pass filter, which consists of OA2, R6, R7, C2 and C3. The output signal is a DC voltage level, which is proportional to the input pulse width.

(a)

Increasing power

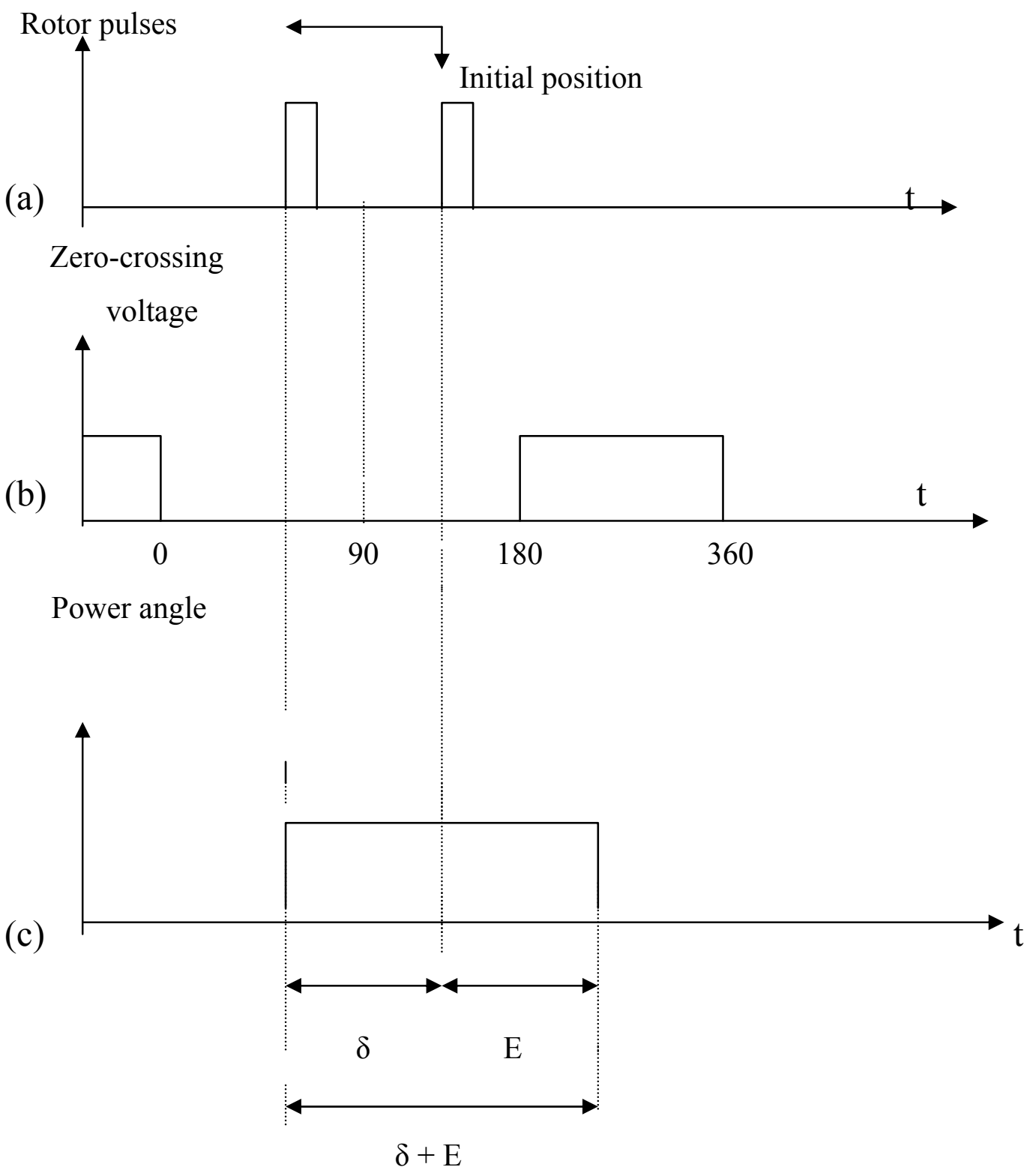

Fig. 4. Pulse waveform for generating the power angle 


\subsection{Error cancellation circuit}

It is well known that the power angle of a synchronous generator is equal to zero at no load condition. Due to the random fixation of the black strip on the rotor axis, there would be some error voltage. The error cancellation circuit cancels this error voltage. The circuit diagram of the error cancellation circuit consists of OA3, OA4, resistors R8, R9, R10, R11, R12, R13 and variable potentiometer VR1. The DC voltages representing the power angle with some error voltage are applied to the non - inverting input of the OA3, which works as a difference amplifier with a gain equal to 10 . The other input of OA3 is a DC voltage representing an error voltage $(E)$, which is subtracted from the composite signal representing power angle and the error $(E)$. The result of the subtraction is a voltage the magnitude of which represents the power angle only. The DC voltage representing the error is obtained from the potential divider consists of VR1 and R13. This error voltage can be adjusted by VR1 to match the error voltage $(E)$, this error signal is applied to the non-inverting input of OA3 through the buffer stage OA4.

\section{ROTOR SPEED MEASURING DEVICE}

As we have mentioned previously, the output pulses from the optical transducer probe are used for generating both power angle and the rotor speed. The rotor speed-measuring device is based on counting of the optical transducer pulses. This device is composed of a single 14-pin I.C. (integrated circuit) type LM 2907 together with some external passive components. This device provides a dc output voltage level proportional to the input frequency and equal to the exact value of the rotor speed $(\dot{\delta})$, it gives a zero output voltage at zero input frequency. The circuit diagram for the rotor speed is shown in figure (5). The integrated circuit used in this device contains an input amplifier with built-in hysteresis, a charge pump frequency to voltage converter, and an operational amplifier with an output transistor.

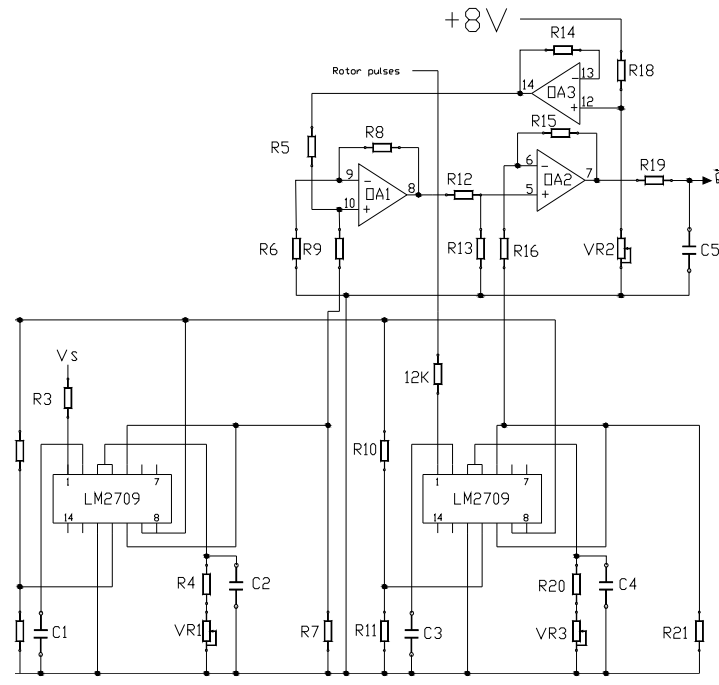

Fig. 5. Circuit diagram of the rotor speed measuring unit 


\section{TERMINAL VOLTAGE MEASURING UNIT}

The principle operation of the voltage transducer is to convert the r.m.s. value of the terminal voltage of the synchronous generator to a $\mathrm{DC}$ voltage level $(\mathrm{Vt})$ suitable to be fed to the A/D converter. The circuit diagram of the voltage transducer is shown in figure (6). The step-down stage is composed of a step-down transformer T1 and a voltage divider composed of R1 and VR1. The attenuated voltage is fed to the full wave precision rectifier (F.W.P.R.) stage, which is composed of a half wave precision that consists of OA1, diodes D1, D2, resistors (R2, R3, R4 and R5) and a scaling subtractor, which is composed of an operational amplifier of OA2, resistors R6 and R7. The output voltage from the F.W.P.R is a train of positive halves of sine waves. These waves are fed to an active low pass filter (L.P.F), which converts these waves to a purely DC voltage level proportional to the R.M.S value of the terminal voltage. The L.P.F is composed of OA3, resistors R8, R9, capacitors C1, $C 2$. The obtained output voltage is fed to the $A / D$ converter through a buffer stage composed of OA4, R10 and R11.

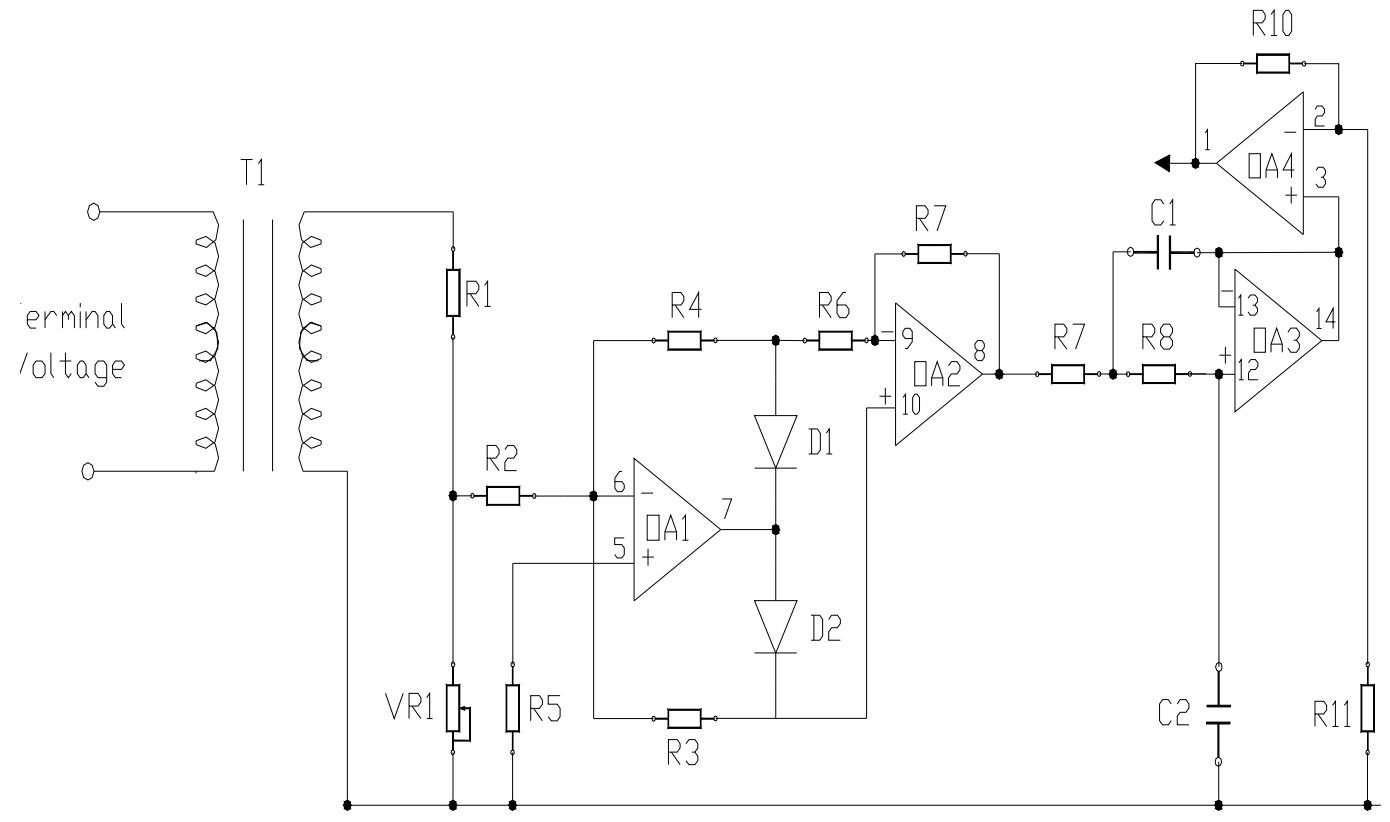

Fig. 6. Circuit diagram of the terminal voltage transducer

\section{PULSES WAVEFORM}

Referring to the circuit diagram of the optical transducer seen in figure (1), the output pulses from the rotor transducer across resistor R5 are shown in figure (7). The time duration between pulses is $20 \mathrm{~ms}$ with $50 \mathrm{~Hz}$ pulse repetition rate and a peak value of $6 \mathrm{~V}$. The pulse width is $2.5 \mathrm{~ms}$, which corresponds to 3000 r.p.m. and $1 \mathrm{~cm}$ width of the black strip.

Referring to the circuit diagram of the power angle transducer seen in figure (5), the differentiated rotor pulses in the non-inverting input of OA1 are shown in figure (8). Also the output pulses obtained from zero crossing detecting circuit of the bus bar voltage (output of COMP1) are shown in figure (9). This output is a train of square pulses of $20 \mathrm{~ms}$ pulse width and $50 \mathrm{~Hz}$ frequencies. Figure (10) shows the output 
pulses obtained from the phase detector circuit (output from $O A_{1}$ ), which represents the pulses of the power angle at no load. In figure (11), the average DC voltage equivalent to the power angle pulses at $70 \%$ of full load is obtained. This DC voltage is the output of the low pass filter OA2. The waveform of this output is a pure DC voltage with minimum amount of ripple. This is because the machine is operating at its steady state operating condition.

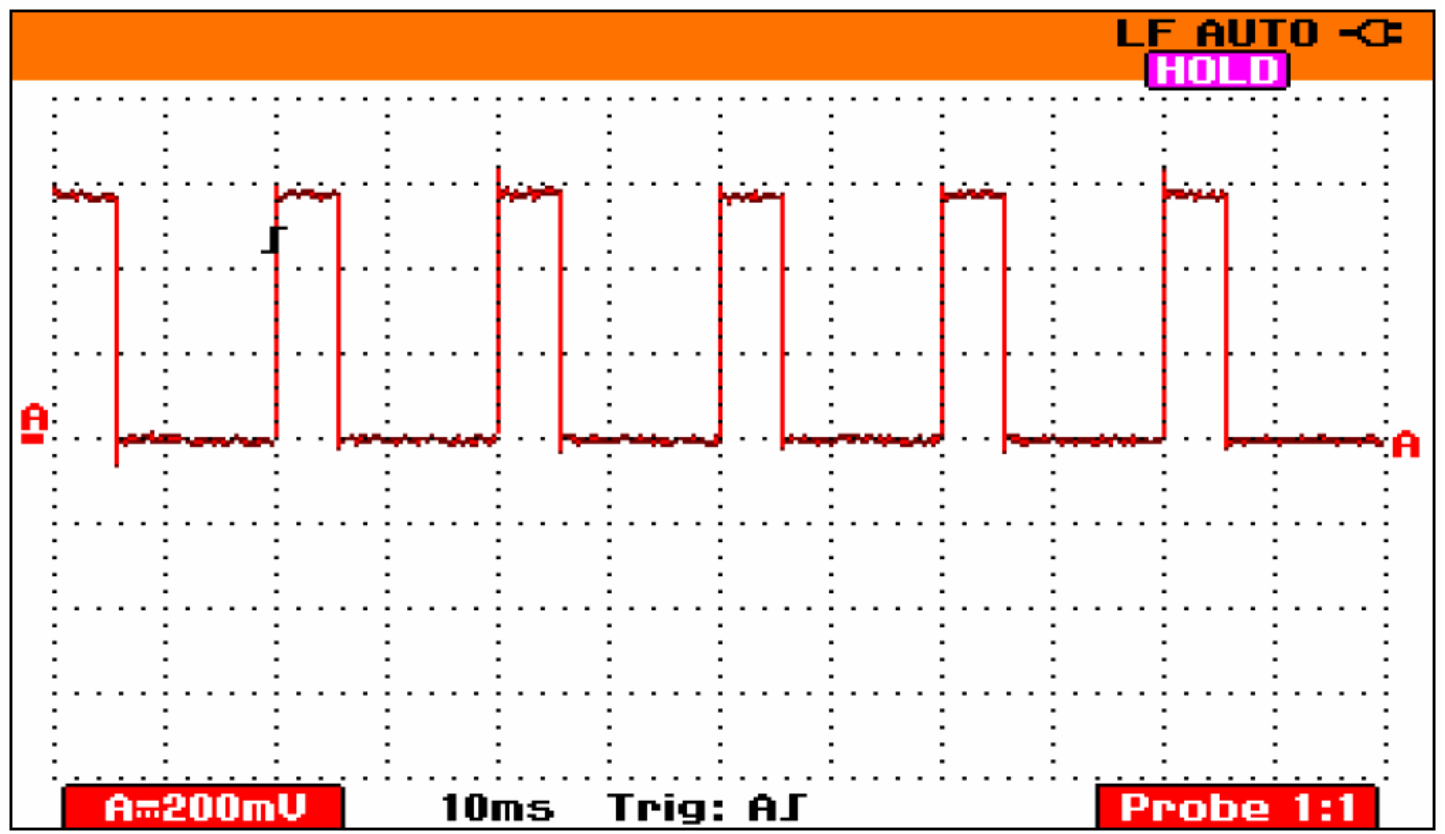

Fig. 7. The output pulses waveforms from the optical rotor transducer

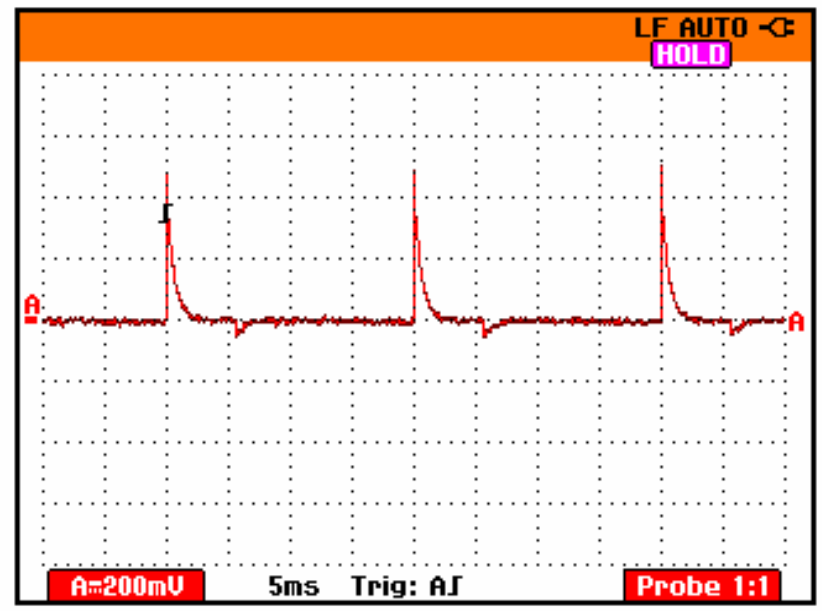

Fig. 8. The differentiated rotor transducer pulses 


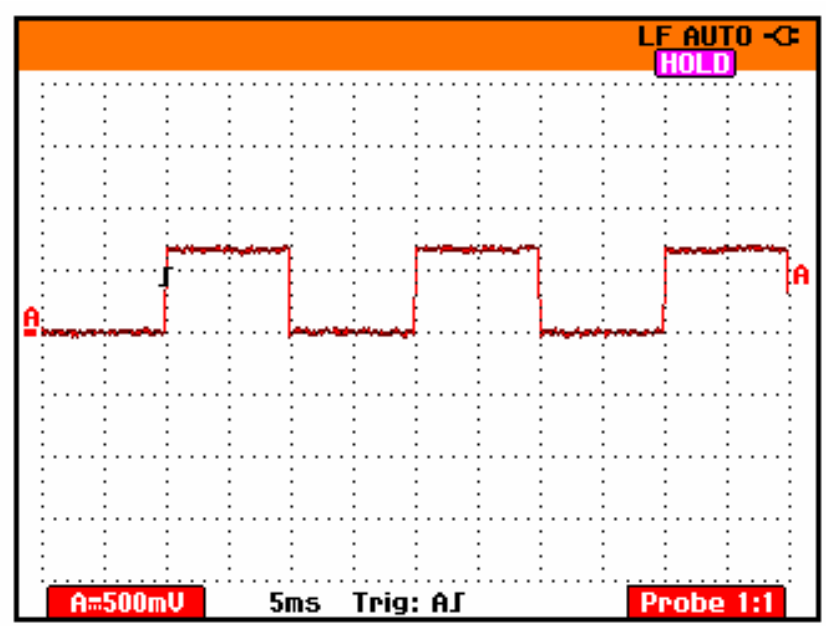

Fig. 9. The output pulses from the zero detecting circuit of the bus bar voltage



Fig. 10. The power angle pulses at no load

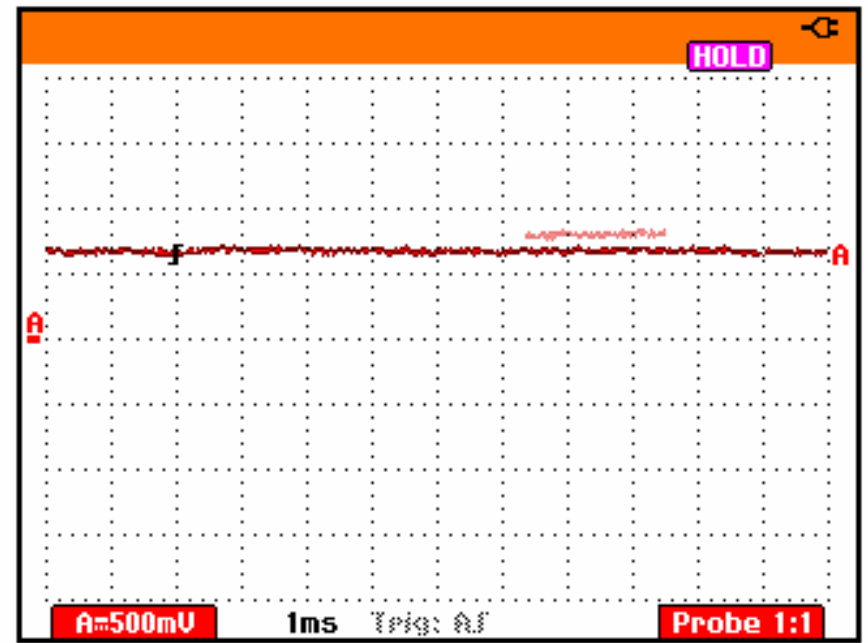

Fig. 11. The DC output voltage equivalent to the power angle 
Figure (12) shows the DC output voltage obtained from the rotor speed transducer. The magnitude of this voltage is proportional to the speed of the prime mover of the synchronous generator. As it can be seen in this figure the obtained voltage is a pure DC wave voltage.

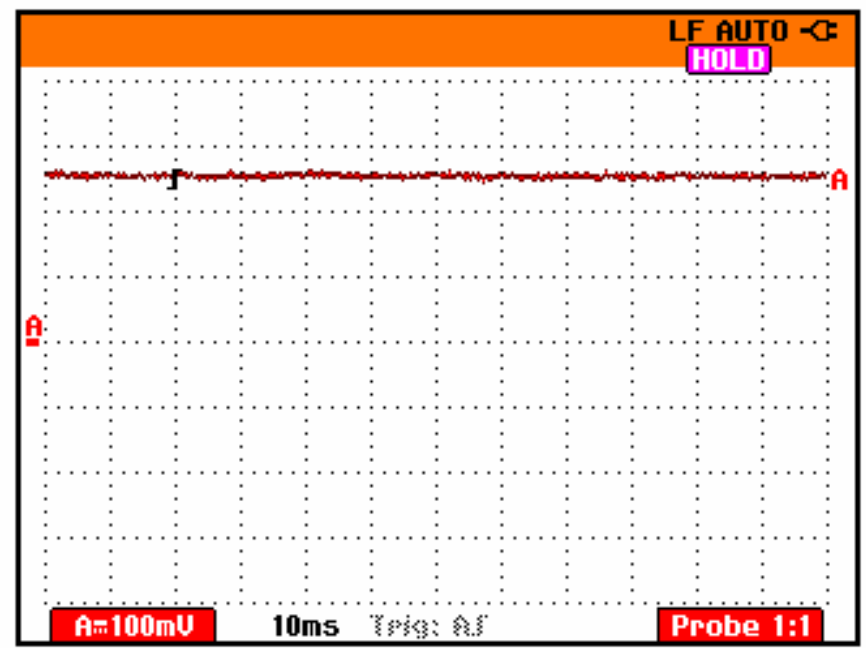

Fig. 12. The DC output voltage equivalent to the rotor speed

Referring to figure (6), which represents the circuit diagram of the terminal voltage transducer $\left(V_{t}\right)$, the output obtained from the full-wave precision rectifier (the output terminal of OA2) is shown in figure (13). The full-wave precision rectifier converts the sine wave signal representing the terminal voltage into accurate full- wave positive halves. This full-wave signal is fed into a low-pass filter in order to convert it into a pure DC voltage, the magnitude of which represents the value of the terminal voltage. The waveform of this signal is shown in figure (14). This DC voltage represents the terminal voltage signal which is fed to the A /D converter through a buffer stage OA4 -figure (6)-.

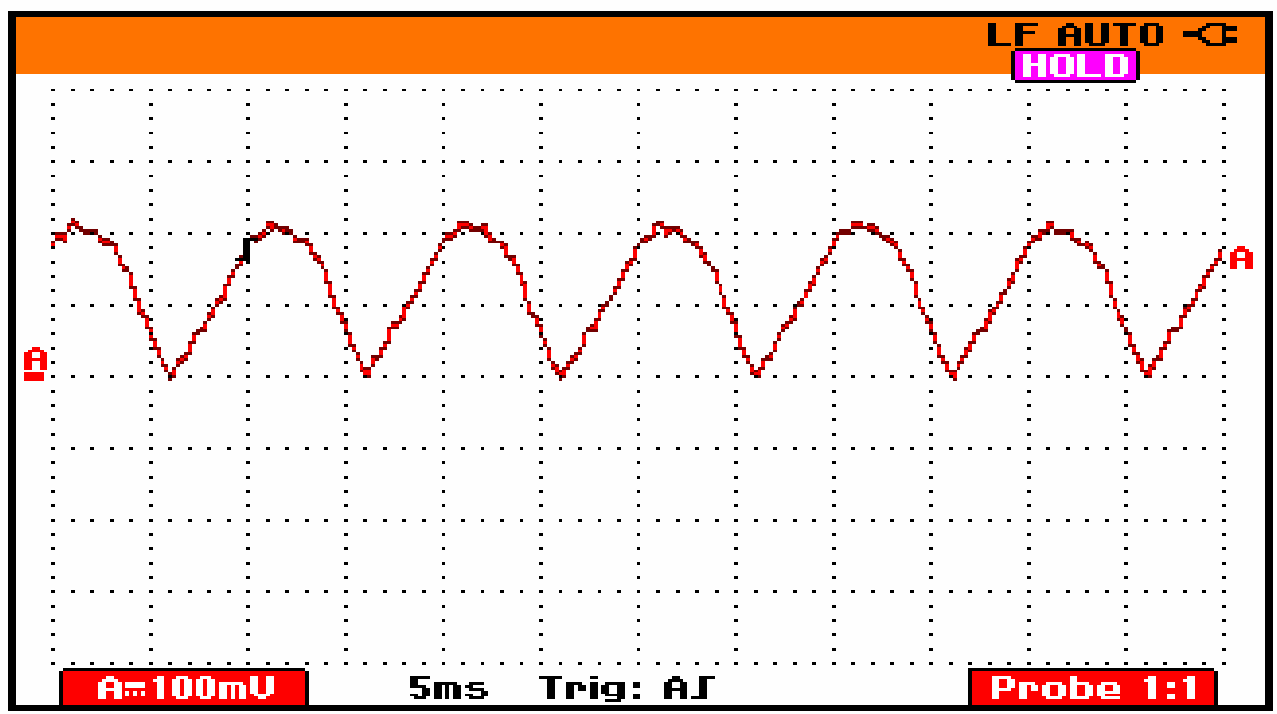

Fig. 13. The output from the full-wave precision rectifier circuit 


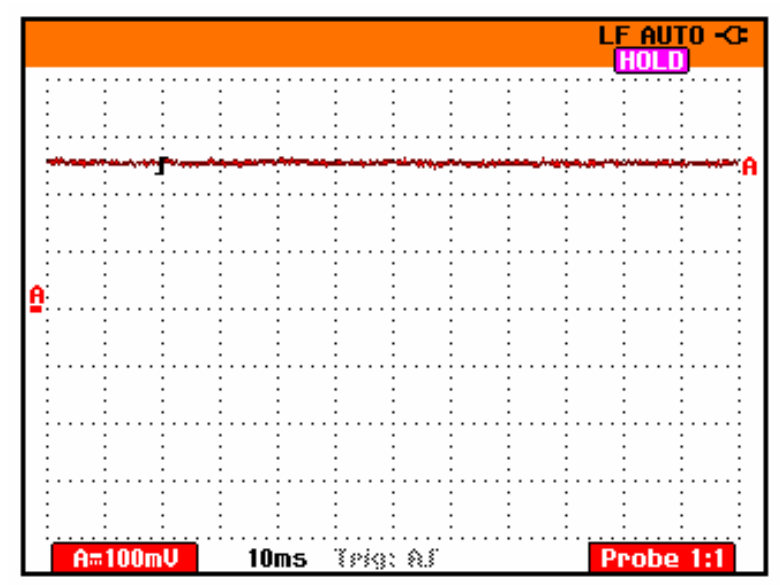

Fig. 14. The output of the low pass filter of the terminal voltage transducer

\section{MEASUREMENT TECHNIQUE}

In order to test the linearity of various electronic transducers, curves between physical input quantities (rotor speed, power angle and terminal voltage) and the transducer output voltage are plotted. The curves reflect the ability of the transducer to produce output voltages similar to these physical input quantities. Figures (15) to (17) illustrate this relation.

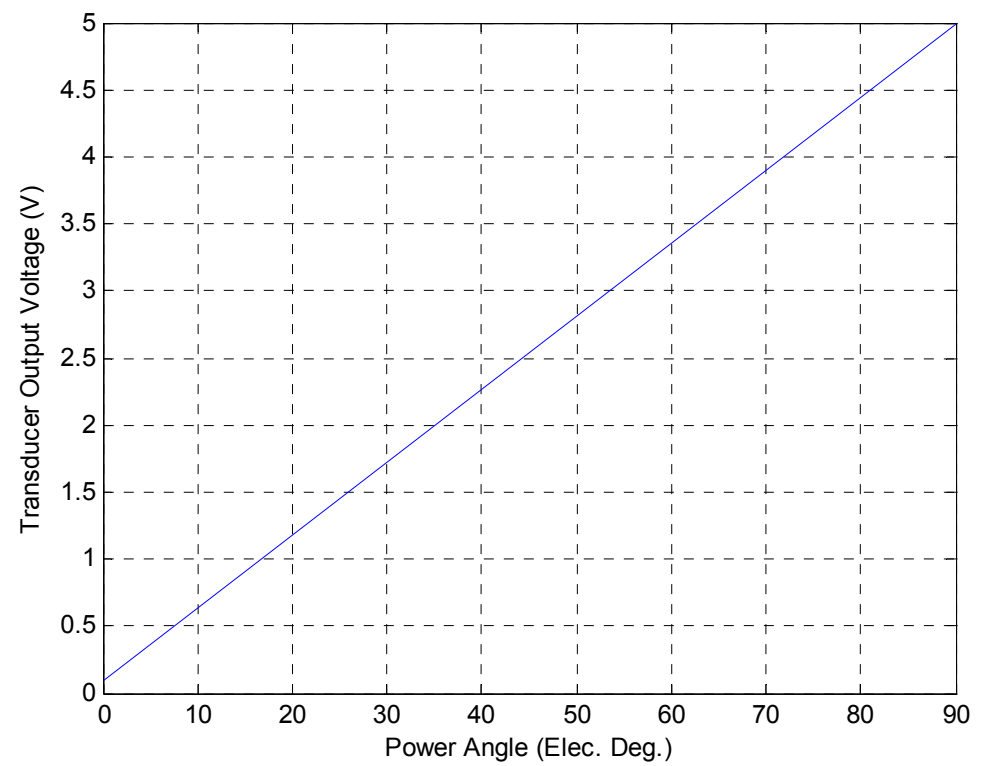

Fig. 15. The power-angle transducer output voltage curve 


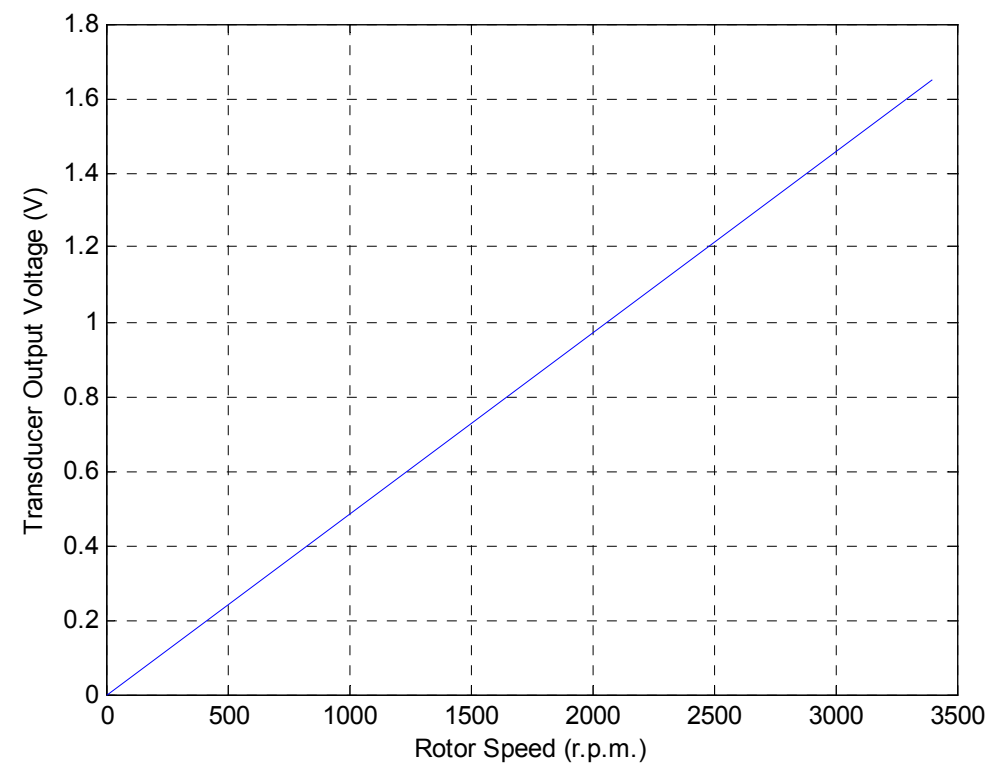

Fig. 16. The rotor speed transducer output voltage curve

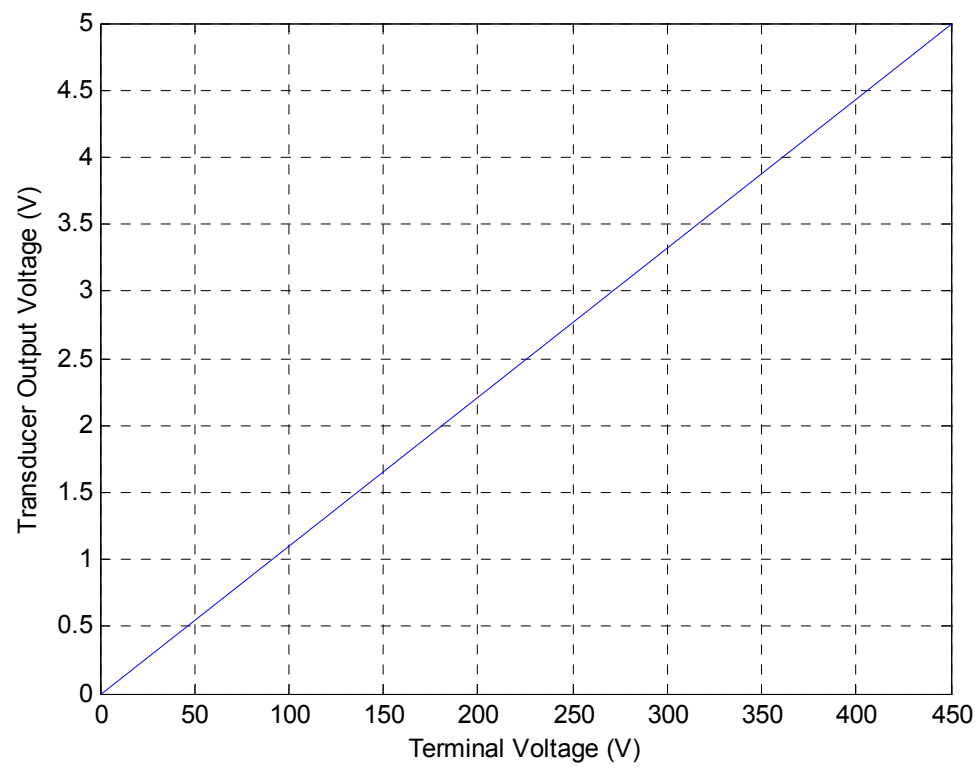

Fig. 17. The terminal voltage transducer output voltage curve

\section{CONCLUSION: MEASUREMENT INSTRUMENT}

The previous ideas were achieved in Electrical Machines Lab. in Military Technical College, Cairo, Egypt, during the period of this research by the article authors. A photograph collection album for the preceding ideas and its laboratory implementation, are presented in the figures (18) to (22). 


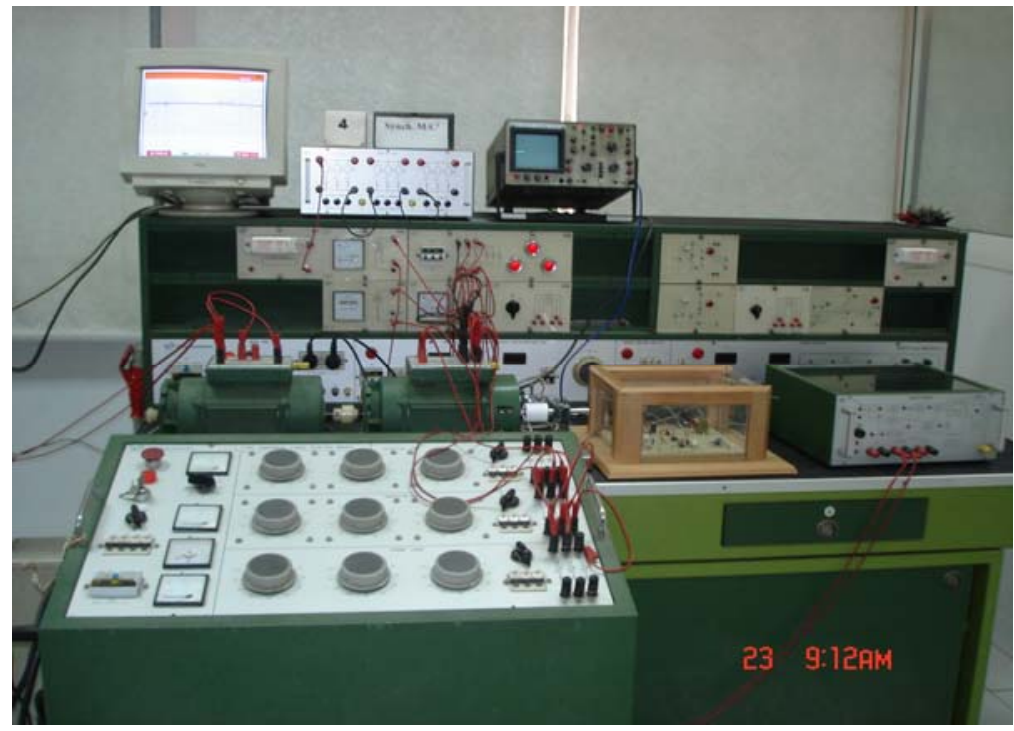

Fig. 18.

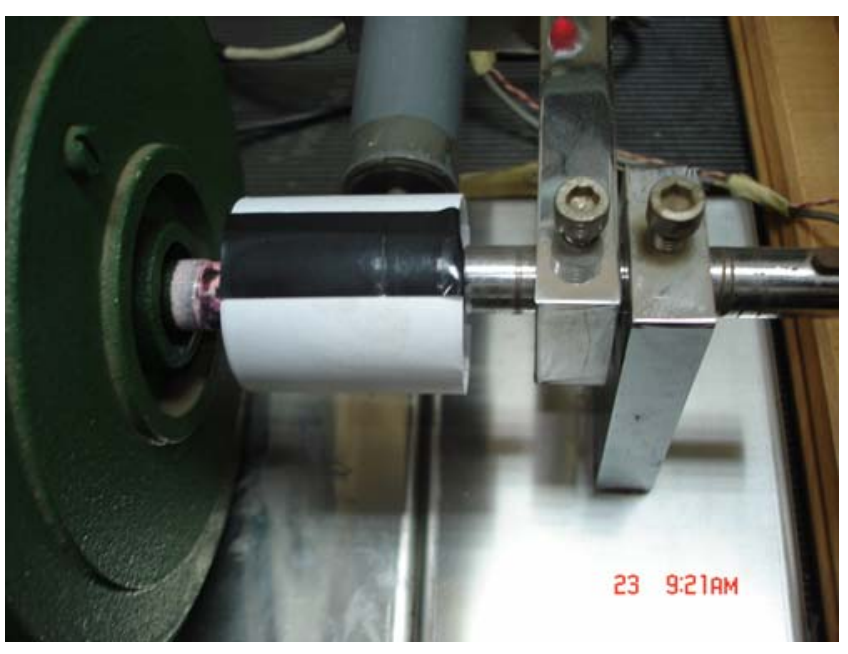

Fig. 19.

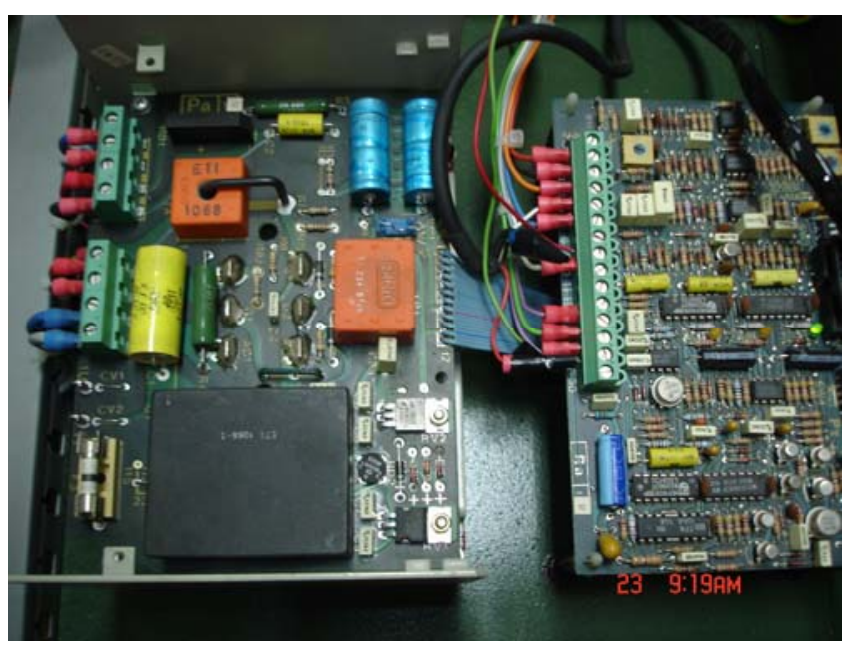

Fig. 21.

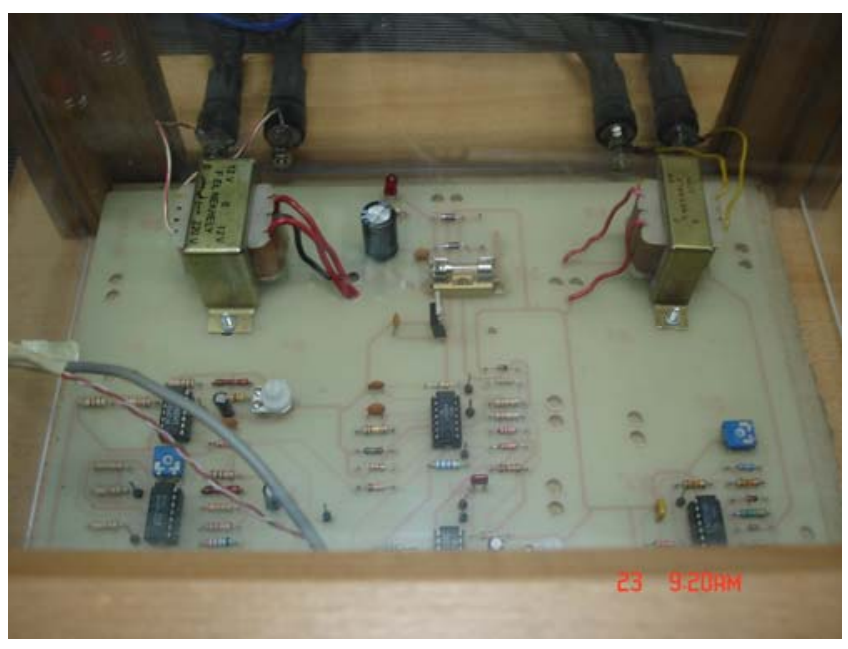

Fig. 20.

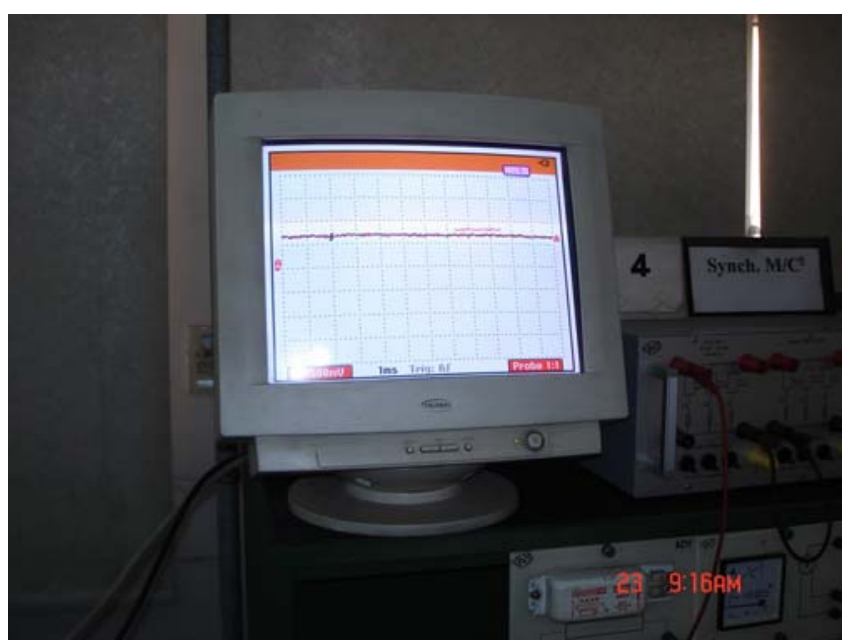

Fig. 22. 


\section{References}

[1] Thorp, J. S., Seyler, C. E. and Phadke, A. G., "Electromechanical Wave Propagation in large Electric Power Systems", IEEE Transactions on Circuits and Systems I: Fundamental Theory and Applications, Vol. 45, No. 6, p 614622, June (1998).

[2] Parashar, M., Thorp, J. S. and Seyler, C. E. "Continuum Modeling of Electromechanical Dynamic in Large-Scale Power Systems", IEEE Transactions on Circuits and Systems I: Regular Papers, Vol. 51, No. 9, p 1848-1858, September (2004).

[3] Phadke, A. G., "Time Synchronizing Techniques, Coupled With The ComputerBased Measurement Techniques, Provide A Novel opportunity to Measure Phasors and Phase Angle Differences In Real Time", IEEE Computer Application in Power, p 10-15, April (1993).

[4] Faulk, D. and Murphy, R. J., "Comanche Peak Unit No. (2), $100 \%$ Load ejection Test Under frequency and System Phasors Measured Across TU Electric system", Proceedings of Annual Conference in Protective Relay Engineers, College Station, TX, March (1994).

[5] Mostafa, R., Khalifa, F. A. and Fekry, M. Y., "A Power Angle and Rotor Speed Measurement Device for Synchronous Machines Stability Study", $4^{\text {th }}$ International Conference on Aeronautical Sciences \& Aviation Technology ASAT 1991, p EE4-623/1-EE4/634, 14-16 May (1991). 